\title{
Correction to: A Perspective Towards NCIFA and CIFA in Named-Data Networking Architecture
}

\author{
Ren-Ting Lee, Yu-Beng Leau, Yong-Jin Park, and Joe H. Obit
}

\section{Correction to:}

Chapter "A Perspective Towards NCIFA and CIFA in Named-Data Networking Architecture" in: R. Alfred et al. (eds.), Computational Science and Technology, Lecture Notes in Electrical Engineering 603, https://doi.org/10.1007/978-981-15-0058-9_46

In the original version of the book, the following post-publication corrections have been incorporated in the chapter 'A Perspective Towards NCIFA and CIFA in Named-Data Networking Architecture':

Correct keywords 'Named-Data Networking, Interest Flooding Attack, Non-Collusive, Collusive Interest Flooding Attack, Collusive, IFA, NCIFA, CIFA, NDN' have been added.

Hyphen has been inserted in the author name 'Yong-Jin Park'.

'Faculty of Computing and Informatics, Universiti Malaysia Sabah, Jalan UMS, Kota Kinabalu, Malaysia' has been added to the affiliation of author Joe H. Obit. Punctuations have been added to References 23 and 31.

The book and chapter have been updated with the changes.

The updated version of this chapter can be found at https://doi.org/10.1007/978-981-15-0058-9_46 